\title{
Development and Characterization of a Collagen-Based Matrix for Vascularization and Cell Delivery
}

\author{
Cara E. Ellis, ${ }^{1,2}$ Laura K. Ellis, ${ }^{1,2}$ Ryan S. Korbutt, ${ }^{1,2}$ Erik J. Suuronen, ${ }^{3}$ and Gregory S. Korbutt ${ }^{1,2, *}$
}

\begin{abstract}
Since the development of the Edmonton protocol, islet transplantation is increasingly encouraging as a treatment for type 1 diabetes. Strategies to ameliorate problems with the intraportal site include macroencapsulating the islets in diverse biomaterials. Characterization of these biomaterials is important to optimally tune the properties to support islets and promote vascularization. In this study, we characterize the cross-linker-dependent properties of collagen-based matrices containing chondroitin-6-sulfate, chitosan, and laminin, cross-linked with 7.5, 30, or $120 \mathrm{mM}$ of 1-ethyl-3-(3-dimethylaminopropyl) carbodiimide and $\mathrm{N}$-hydroxysuccinimide. The swelling ratio was found to be significantly negatively correlated with increasing cross-linker concentrations $(p<0.0001 ; R 2=0.718)$. The matrix released insulin in a reproducible logarithmic manner (R2 of 0.99 for all concentrations), demonstrating cross-linker-dependent control of drug release. The matrices with the highest cross-linker concentrations resisted degradation by collagenase for longer than the lowest concentrations $(58.13 \% \pm 2.22 \%$ vs. $13.69 \% \pm 7.67 \% ; p<0.05)$. Scanning electron microscopy images of the matrices revealed that the matrices had uniform topography and porosity, indicating efficient cross-linking and incorporation of the polymer components. Matrices were transplanted subcutaneously in naive BALB/C mice, and the number and size of vessels were quantified using von Willebrand factor staining; matrices with higher cross-linking concentrations had significantly larger capillaries at every time point up to 4 weeks after transplantation compared to the lowest cross-linker concentration group. CD31 staining visualized the capillaries at each time point. Taken together, these data show that this collagen-based matrix is reproducible with cross-linking-dependent properties that can be optimized to support vascularization and islet function.
\end{abstract}

Key words: angiogenesis; biomaterials; extracellular matrix; tissue engineering

\section{Introduction}

Islet transplantation is a promising clinical cell-based therapy for treatment of type 1 diabetes. ${ }^{1-4}$ However, despite remarkable progress, the liver implantation site remains far from ideal. Clinical transplantation of islets into the portal vein has been associated with life-threatening intraperitoneal bleeding, ${ }^{5}$ portal vein thrombosis, and hepatic steatosis. ${ }^{6,7}$ The liver may also contribute to the gradual attrition of chronic islet graft function. ${ }^{8}$ Search for a safer alternative site for islet transplantation is therefore desirable and an important issue to address. ${ }^{9}$ Using biomaterials to deliver islets to an alternate site could be advantageous if vascularization was promoted, particularly if an immune barrier could also be incorporated into the device. ${ }^{9}$ Diverse techniques have been attempted to this end, including utilizing a polyethylene terephthalate mesh bag, ${ }^{10}$ a polyurethane foam dressing, ${ }^{11}$ a stainless steel mesh with polytetrafluoroethylene stoppers, ${ }^{12}$ and gelatin microspheres in a collagen-coated polyvinyl bag. ${ }^{13}$

${ }^{1}$ Department of Surgery and ${ }^{2}$ Alberta Diabetes Institute, University of Alberta, Edmonton, Canada.

${ }^{3}$ Division of Cardiac Surgery, University of Ottawa Heart Institute, Ottawa, Canada.

*Address correspondence to: Gregory S. Korbutt, PhD, Alberta Diabetes Institute, University of Alberta, 5-002 Li Ka Shing Centre for Health Research Innovation, Edmonton, AB, T6G 2E1 Canada, E-mail: korbutt@ualberta.ca

(C) Cara E. Ellis et al. 2015; Published by Mary Ann Liebert, Inc. This Open Access article is distributed under the terms of the Creative Commons License (http://creativecommons.org/licenses/by/4.0), which permits unrestricted use, distribution, and reproduction in any medium, provided the original work is properly credited. 
Most devices for islet delivery are based on synthetic polymers, which offer the advantages of complete control over mechanical and chemical properties and lower manufacturing $\operatorname{costs}{ }^{14,15}$; however, natural polymers offer significant advantages of their own, including the ability of their degradation by-products to be metabolized. ${ }^{14,15}$ In addition, these natural polymers can be engineered to mimic the properties of the natural extracellular matrix (ECM) to support various cell types, including islets and recruited recipient cells. ${ }^{16}$

Collagen-based biomaterial matrices have been previously used to deliver neonatal porcine islets (NPIs) subcutaneously in a murine model. ${ }^{17,18}$ This matrix contains the copolymers chondroitin-6sulfate, chitosan, and laminin and is cross-linked with 1-ethyl-3-(3-dimethylaminopropyl) carbodiimide and $\mathrm{N}$-hydroxysuccinimide to support NPI viability and function. The matrix is formed in a planar shape to improve graft oxygenation ${ }^{10}$ and has sufficient mechanical strength to resist the mechanical stress of the subcutaneous site. This is an attractive approach to creating a highly vascularized site for the implantation of islets, particularly because the matrix could be functionalized with growth factors that promote angiogenesis. ${ }^{10}$ Our work has demonstrated that this matrix has no effect on glucose-stimulated insulin secretion and can support NPI viability and function in vivo. ${ }^{17,18} \mathrm{It}$ is important to characterize any biomaterial to ensure that the material functions in a way that supports the viability and function of the target cells; for a material that is intended for vascularization, these properties would include degradation rate, swelling ratio, and degree of vascularization. Biocompatibility depends not only on the material characteristics but also on the biological system in which the material will be used, and therefore, it is valuable to be able to alter the material properties to suit the applications. ${ }^{19}$ Natural polymers can be tuned by various strategies, including the use of copolymers and controlling the degree of cross-linking of all the polymers. ${ }^{15}$ Additionally, it is known that the topography of a material is important as an alternate signaling mechanism to control many properties related to vascularization, including adhesion, migration, and differentiation. ${ }^{15,16}$ We have previously optimized the copolymers in our collagenbased matrix, ${ }^{18,20,21}$ but the most favorable concentration of the cross-linker remains to be determined. In this study, the cross-linker-dependent properties of the collagen-based matrix are characterized to facilitate the optimization of a biomaterial able to support cellular grafts, such as islets for the treatment of type 1 diabetes.

\section{Materials and Methods}

NPI preparation

Donor pancreases were obtained from 1- to 3-day-old Duroc cross neonatal piglets from the University of Alberta Swine Research Centre $(1.5-2.0 \mathrm{~kg}$ body weight), and the islets were isolated and cultured for 5-7 days, as described previously. ${ }^{18,22}$ Briefly, the retrieved pancreases were cut into 1 - to $3-\mathrm{mm}$ tissue fragments, then exposed to $2.5 \mathrm{mg} / \mathrm{mL}$ collagenase (type XI, C7657; Sigma), filtered through a $500-\mu \mathrm{m}$ nylon screen, and washed in Hank's Basic Salt Solution (HBSS, H6136; Gibco) supplemented with 0.25\% BSA (fraction V, A9543; Sigma-Aldrich), $10 \mathrm{mM}$ HEPES (H4034; Sigma-Aldrich), $100 \mathrm{U} / \mathrm{mL}$ penicillin, and $0.1 \mathrm{mg} / \mathrm{mL}$ streptomycin (09-757F; Lonza Walkersville, Inc.). NPI were then cultured in nontissue culture-treated Petri dishes containing Ham's F10 tissue culture media (N6635; Sigma-Aldrich) supplemented with $14.3 \mathrm{mM}$ sodium bicarbonate (S233; Fisher), $10 \mathrm{mM}$ D-glucose (DX0145-3; EM Science), 2 mM L-glutamine (G8540; Sigma-Aldrich), $0.25 \%$ BSA (fraction V), $50 \mu \mathrm{M}$ isobutylmethylxanthine (I5879; Sigma-Aldrich), $10 \mathrm{mM}$ nicotinamide (N0636; Sigma-Aldrich), $1.6 \mathrm{mM}$ calcium chloride dihydrate (C7902; Sigma-Aldrich), $100 \mathrm{U} / \mathrm{mL}$ penicillin, and $0.1 \mathrm{mg} / \mathrm{mL}$ streptomycin $(09-757 \mathrm{~F}$; Lonza Walkersville, Inc.). The islets were cultured at $37^{\circ} \mathrm{C}$ for $5-7$ days, with the medium changed at the first, third, and fifth days after isolation.

\section{Preparation of collagen matrices}

Collagen matrices cross-linked with 7.5, 30, and $120 \mathrm{mM}$ of 1-ethyl-3-(3-dimethylaminopropyl) carbodiimide (EDC, E6383; Sigma-Aldrich) and N-hydroxysuccinimide (56480; Sigma-Aldrich), containing $0.2 \mathrm{mg} / \mathrm{mL}$ chondroitin-6-sulfate (034-14612; Wako Pure Chemical Industries), $1.0 \mathrm{mg} / \mathrm{mL}$ chitosan (C3646; SigmaAldrich), and $0.1 \mathrm{mg} / \mathrm{mL}$ mouse laminin (354232; BD Biosciences, Inc.), were manufactured using previously described methods. ${ }^{18,22}$ High-concentration denatured rat tail type 1 collagen (354249; BD Biosciences, Inc.) was used. Briefly, all components were mixed together in a $50-\mathrm{mL}$ glass tube on ice, with varying concentrations of cross-linker added at the last time. The liquid matrix was then adjusted to a $\mathrm{pH}$ of 6.0 for $5 \mathrm{~min}$ to initiate cross-linking, then $150 \mu \mathrm{L}$ of liquid matrix was added to the wells of a 24-well plate; matrices were then cross-linked for $30 \mathrm{~min}$ at $37^{\circ} \mathrm{C}$ before continuing 
on to the in vitro or in vivo analyses. Before transplantation, matrices were cultured for $24 \mathrm{~h}$ in phosphatebuffered saline (PBS) to ensure excess cross-linker was removed.

\section{In vitro measurements of matrix properties}

The masses of the matrices were measured after a 30 min cross-linking period. The matrices were then dehydrated for $1 \mathrm{~h}$ in increasing concentrations of ethanol, from $70 \%$ to $100 \%$, in periods of $15 \mathrm{~min}$. The masses of the fully dehydrated matrices were measured three times to ensure consistency throughout the test. The swelling ratio $(\mathrm{Q})$ of the matrix was calculated by the following formula:

$$
Q=\frac{M_{c}-M_{d}}{M_{c}}
$$

where $M_{c}=$ cross-linked weight and $M_{d}=$ dehydrated weight.

To ensure that hormones or growth factors can readily diffuse out of the matrix, $34.7 \mu \mathrm{g}$ (1 UI) of porcine insulin was added to $1000 \mu \mathrm{L}$ of liquid matrix in triplicate and then PBS without insulin was added on top of the matrices. All the PBS was removed, and fresh PBS was added at $30 \mathrm{~min}, 1 \mathrm{~h}, 2 \mathrm{~h}, 3 \mathrm{~h}, 4 \mathrm{~h}$, and $6 \mathrm{~h}$ to mimic biologically fast acting insulin. The PBS was subsequently assayed for porcine insulin using a commercial mouse/ rat insulin assay (K152BZC; Meso Scale Diagnostics).

\section{Scanning electron microscopy}

Matrices were also taken for scanning electron microscopy (SEM) analysis of the microstructure using the technique described by McEwan et al. ${ }^{20}$ Briefly, to preserve cell morphology, matrices with cells were fixed in 3\% glutaraldehyde (Sigma-Aldrich) buffered with $0.1 \mathrm{M}$ PBS for $30 \mathrm{~min}$ and then rinsed with PBS three times before ethanol washes. Ten millimeters of diameter matrix samples were dehydrated in $70 \%, 80 \%$, $90 \%, 95 \%$, and $100 \%$ ethanol solutions for $10 \mathrm{~min}$ each. For cross-sectional viewing, samples were fractured after immersion in liquid nitrogen. Samples were sputtered (Hummer VII; Anatech) with a palladium/gold (60:40 palladium:gold) alloy to form a thin coating $(3 \mathrm{~nm})$. SEM images were obtained using an accelerating voltage of $1.0 \mathrm{kV}$ to minimize sample damage. Cross-sectional images were obtained using backscattering and secondary electron detectors. Micrographs were evaluated for porosity and pore diameter using ImageJ $1.43 \mathrm{u}$ software.

\section{In vitro degradation}

The masses of matrices with 7.5, 30, and $120 \mathrm{mM}$ crosslinker concentrations were measured, and then, the matrices were exposed to $25 \mathrm{U}$ of type $\mathrm{V}$ collagenase in $\mathrm{PBS}$ at $37^{\circ} \mathrm{C}$ ( $n=3$ for each matrix type). At $30 \mathrm{~min}$, $1 \mathrm{~h}$, then hourly up to $10.5 \mathrm{~h}$, the collagenase solution was aspirated and any adsorbed solution removed, and then, the matrices were weighed to determine the in vitro degradation rate.

\section{NPI survival}

Apoptosis of the NPIs in the matrices was assessed after 7 days in culture using a commercial TUNEL assay kit (A23210; Invitrogen Molecular Probes). The matrices were fixed in Z-Fixx, paraffin embedded, and then, slides of sections were prepared. After rehydration, antigen retrieval was performed in a sodium citrate buffer ( $\mathrm{pH}$ 6.0), and then, slides were incubated according to the manufacturer's instructions. The slides were mounted with ProLong Gold Antifade Reagent with DAPI (P36935; Invitrogen Molecular Probes). To quantify the percentage of TUNELpositive cells, five images of TUNEL and DAPI staining were taken, and then, the images were combined in ImageJ (National Institutes of Health). Separate images of the TUNEL- and DAPI-positive channels were altered to black and white, and then, the number of particles was counted. Each particle was confirmed by two independent reviewers to be TUNEL or DAPI positive visually and the brightness of the color threshold adjusted to only include positive cells. The number of TUNEL-positive cells was subsequently divided by the total DAPI-positive cells to determine the percentage of apoptotic cells in each image.

\section{In vivo measurements of matrix properties}

Matrices with varying cross-linker concentrations were transplanted subcutaneously under the dorsal skin of naive $\mathrm{BALB} / \mathrm{c}$ mice. These matrices were retrieved at 2, 3, and 4 weeks. After retrieval, all collagen-based matrices were fixed in Shandon Zinc Formal-Fixx (6764255; Thermo Fisher Scientific), then embedded in paraffin, and $5-\mu \mathrm{m}$ sections were prepared. For vascularization, CD31 and von Willebrand factor (vWF) staining was utilized to visualize arterioles and capillaries. After rehydration, antigen retrieval for both CD31 and vWF was performed in the sodium citrate buffer (pH 6.0). All immunohistochemical samples were blocked with $20 \%$ normal goat serum for $20 \mathrm{~min}$ (0005-000121; Jackson ImmunoResearch Laboratories, Inc.). 
Slides were visualized with an Axioscope II microscope equipped with an AxioCam MRC and analyzed with Axiovision 4.6 software (Carl Zeiss). Five images of vWF staining were taken from each of two retrieved matrices for each cross-linker concentration, and the images were combined for particle analysis in ImageJ (National Institutes of Health). Vessels were counted if they were between 50 and $10,000 \mu \mathrm{m}^{2}$ in size, with a circularity greater than 0.2 . Each particle was confirmed to be a capillary-like structure visually and excluded manually if mistakenly included by the software. Images of CD31 staining were also obtained to confirm that the vWF staining identifies the quality and stability of the vessels.

\section{Results}

Swelling behavior and drug release

are cross-linker dependent

The swelling ratio of the collagen-based matrix was significantly correlated with the cross-linking concentration $(p<0.0001)$, with a coefficient of determination of 0.718 (Fig. 1A). Increasing the cross-linking concentration resulted in a lower swelling ratio, consistent with hydrogel behavior.

Insulin was diffused from the matrix with all crosslinking concentrations in a reproducible logarithmic manner, with a coefficient of determination of 0.99 for all concentrations (Fig. 1B). By the end of $6 \mathrm{~h}$, a total of $30.62 \mu \mathrm{g}$ of insulin was released from the matrices with $7.5 \mathrm{mM}$ cross-linker ( $88 \%$ of the total insulin), $27.89 \mu \mathrm{g}$ of insulin had been released from the matrices with $30 \mathrm{mM}$ cross-linker or $\sim 80 \%$ of the total insulin, and $28.90 \mu \mathrm{g}$ of insulin had been released from the matrices with $120 \mathrm{mM}$ cross-linker (83\% of total). A lower crosslinker concentration seemed to release insulin more quickly, but none of the groups was significantly different.

Increased cross-linker concentration

gives resistance to in vitro degradation

The matrices with $7.5 \mathrm{mM}$ cross-linking concentration were significantly more degraded as early as $30 \mathrm{~min}$ compared to the matrices with 30 and $120 \mathrm{mM}$ crosslinking concentrations $(77.20 \% \pm 3.73 \%$ vs. $90.41 \% \pm$ $0.57 \%$ and $86.76 \% \pm 1.64 \%$, respectively; $p<0.001$; Fig. 1C). The $7.5 \mathrm{mM}$ cross-linking concentration matrices were also significantly more degraded at $1 \mathrm{~h}$ compared to the matrices with $30 \mathrm{mM}$ and $120 \mathrm{mM}$ cross-linkers $(43.32 \% \pm 2.59 \%$ vs. $86.32 \% \pm 1.63 \%$ and $80.73 \% \pm 4.12 \%$, respectively; $p<0.01)$ and at $2 \mathrm{~h}(18.62 \% \pm 0.23 \%$ vs. $72.12 \% \pm 6.65 \%$ and $69.52 \% \pm 4.56 \% ; p<0.01)$. These low cross-linking concentration matrices were completely degraded after $3 \mathrm{~h}$ of exposure to $25 \mathrm{U} / \mathrm{mL}$ collagenase. There were no significant differences in degradation between the matrices with 30 and $120 \mathrm{mM}$ cross-linking concentrations at any time point; both groups were too degraded to be measured after $10.5 \mathrm{~h}$.

Increased cross-linker concentration is associated with increased vascularization

At 2 weeks after transplantation, there were no significant differences between the capillary sizes in the matrices with $7.5,30$, or $120 \mathrm{mM}$ cross-linker concentrations $(p=0.054$; Fig. 1D). At 3 weeks, the matrix with $120 \mathrm{mM}$ cross-linker concentration had significantly larger capillaries $(270.38 \pm 4.89 \mu \mathrm{m})$ compared to the other two cross-linker concentrations (50.82 \pm 1.52 and $75.24 \pm 2.32 \mu \mathrm{m}$ for 7.5 and $30 \mathrm{mM}$ crosslinker concentrations, respectively; $p<0.05)$ and compared to the mean size of the capillaries at 2 weeks. Additionally, the matrix with $30 \mathrm{mM}$ cross-linker concentration had a significantly larger mean vessel size compared to the matrices with $7.5 \mathrm{mM}$ cross-linker concentration. Similarly, at 4 weeks, the matrix with $120 \mathrm{mM}$ cross-linker concentration had significantly larger capillaries $(433.12 \pm 5.12 \mu \mathrm{m})$ compared to the other two cross-linker concentrations $(81.82 \pm 1.20$ and $221.68 \pm 5.63 \mu \mathrm{m}$ for 7.5 and $30 \mathrm{mM}$ cross-linker concentrations, respectively; $p<0.05)$ and compared to the mean size of the capillaries at 2 weeks. Again, the matrices with $30 \mathrm{mM}$ cross-linker concentration had a significantly larger mean capillary size compared to the $7.5 \mathrm{mM}$ cross-linker concentration matrices $(p<0.05)$. All groups had significantly larger capillaries than the earlier time points. These quantitative data are supported by CD31 staining. Two weeks after transplantation, CD31-positive cells and some small vessels can be observed in the matrices with $7.5 \mathrm{mM}$ (Fig. 2A), $30 \mathrm{mM}$ (Fig. 2B), and $120 \mathrm{mM}$ (Fig. 2C) cross-linker concentrations. As the cross-linking concentration increases, larger vessels are visible. Four weeks posttransplantation, all matrices exhibited many CD31positive vessels, with the matrices with $120 \mathrm{mM}$ cross-linker concentration showing the largest and most plentiful vessels (Fig. 2F) compared to those with $7.5 \mathrm{mM}$ (Fig. 2D) and $30 \mathrm{mM}$ (Fig. 2E) crosslinker concentrations.

Nanotopography is cross-linker dependent SEM analysis revealed that the matrix had uniform topography and porosity, indicating efficient cross-linking 

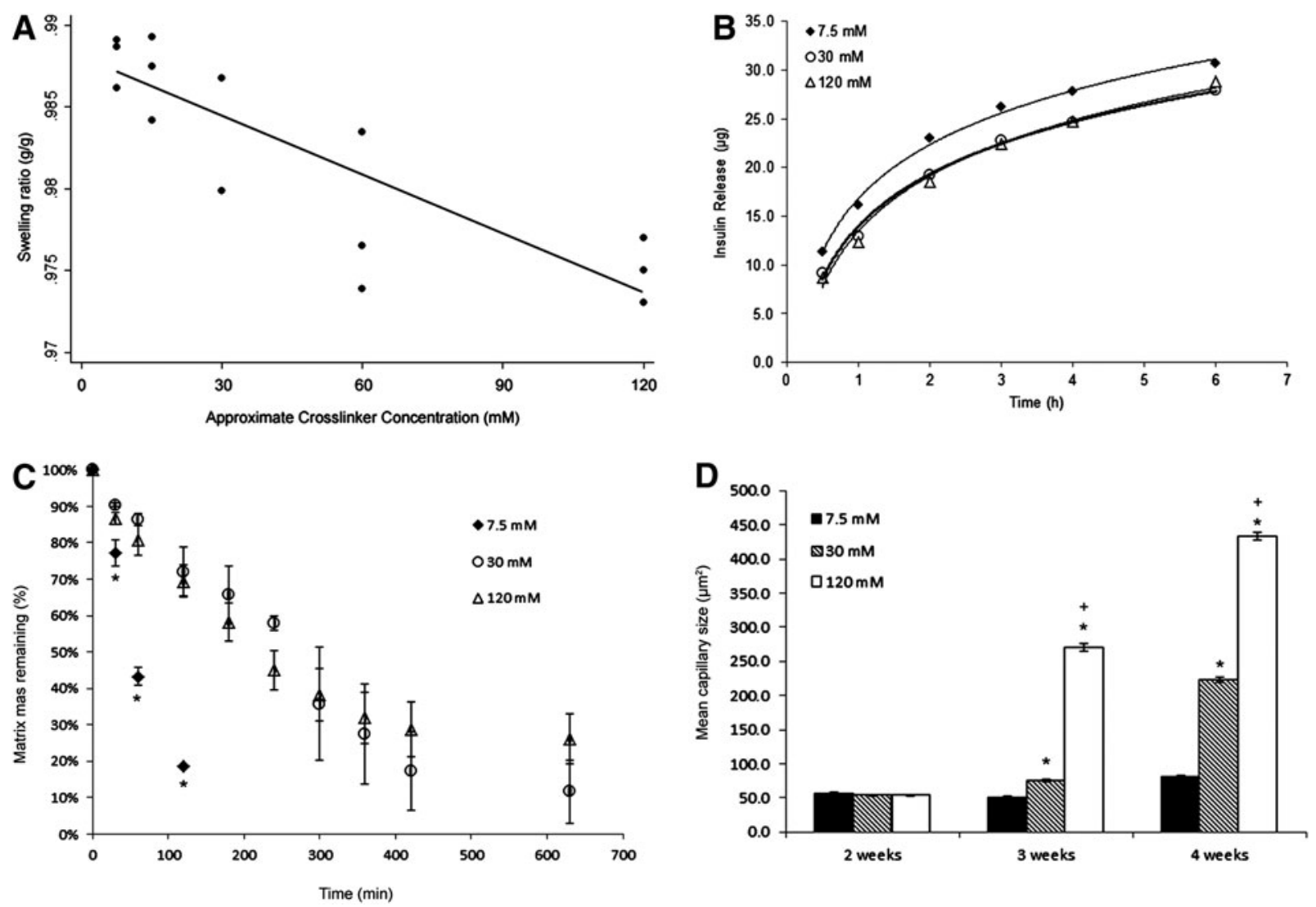

FIG. 1. (A) Matrices were weighed immediately after the cross-linking period and then dehydrated in increasingly concentrated ethanol solutions. The swelling ratio was calculated as the ratio of the difference between the cross-linked matrix mass and the dehydrated matrix mass to the cross-linked matrix mass. (B) One unit of insulin was added to matrices with 7.5, 30, or $120 \mathrm{mM}$ cross-linker concentration, and then, PBS was added on top of the matrices. The insulin diffusion into the PBS was measured at 30 min, then at 1, 2, 3, 4, and $6 \mathrm{~h}$. (C) Matrices were exposed to $25 \mathrm{U}$ of type $\mathrm{V}$ collagenase. The masses of the matrices were measured at $30 \mathrm{~min}$, then hourly up to $10 \mathrm{~h}$, or until the matrices were completely degraded. (D) Matrices were transplanted subcutaneously in naive BALB/c mice and then retrieved at 2, 3, or 4 weeks after transplantation. Matrices were paraffin embedded, then the sections were stained for vWF, and the capillary size was measured using ImageJ. * $p<0.05$ versus $7.5 \mathrm{mM}$ cross-linking concentration; ${ }^{+} p<0.05$ versus $30 \mathrm{mM}$ cross-linking concentration. All results are shown as mean \pm standard error of mean. PBS, phosphate-buffered saline; vWF, von Willebrand factor.

and incorporation of the different components. At a micrometer level, geometric changes in the surface of the matrices were observed. Matrices with $7.5 \mathrm{mM}$ cross-linking concentration were observed to have micropores with a diameter of $166 \pm 6.4 \mu \mathrm{m}$ (Fig. 3A), whereas matrices with $120 \mathrm{mM}$ cross-linking had microconvexities with a diameter of $148 \pm 8.0 \mu \mathrm{m}$ (Fig. 3C). The nanotopography of the surface of the matrices did not vary with cross-linking concentrations and appeared to be very smooth for a depth of $50 \pm$
$4.3 \mathrm{~nm}$. Below this smooth surface, a randomized network of cross-links was visible. The matrices with $7.5,30$, and $120 \mathrm{mM}$ cross-linking concentrations had significantly different average crosslink densities of $63 \% \pm 2.2 \%, 70 \% \pm 2.5 \%$, and $82 \% \pm 2.3 \%$, respectively $(p<0.05$ between 120 and $30 \mathrm{mM}$, and 30 and $7.5 \mathrm{mM} ; p<0.01$ between 120 and $7.5 \mathrm{mM}$; Fig. $3 \mathrm{D}-\mathrm{F})$. The average cross-link diameters of all three matrices were not significantly different (137.8 \pm $12.0 \mathrm{~nm})$. 


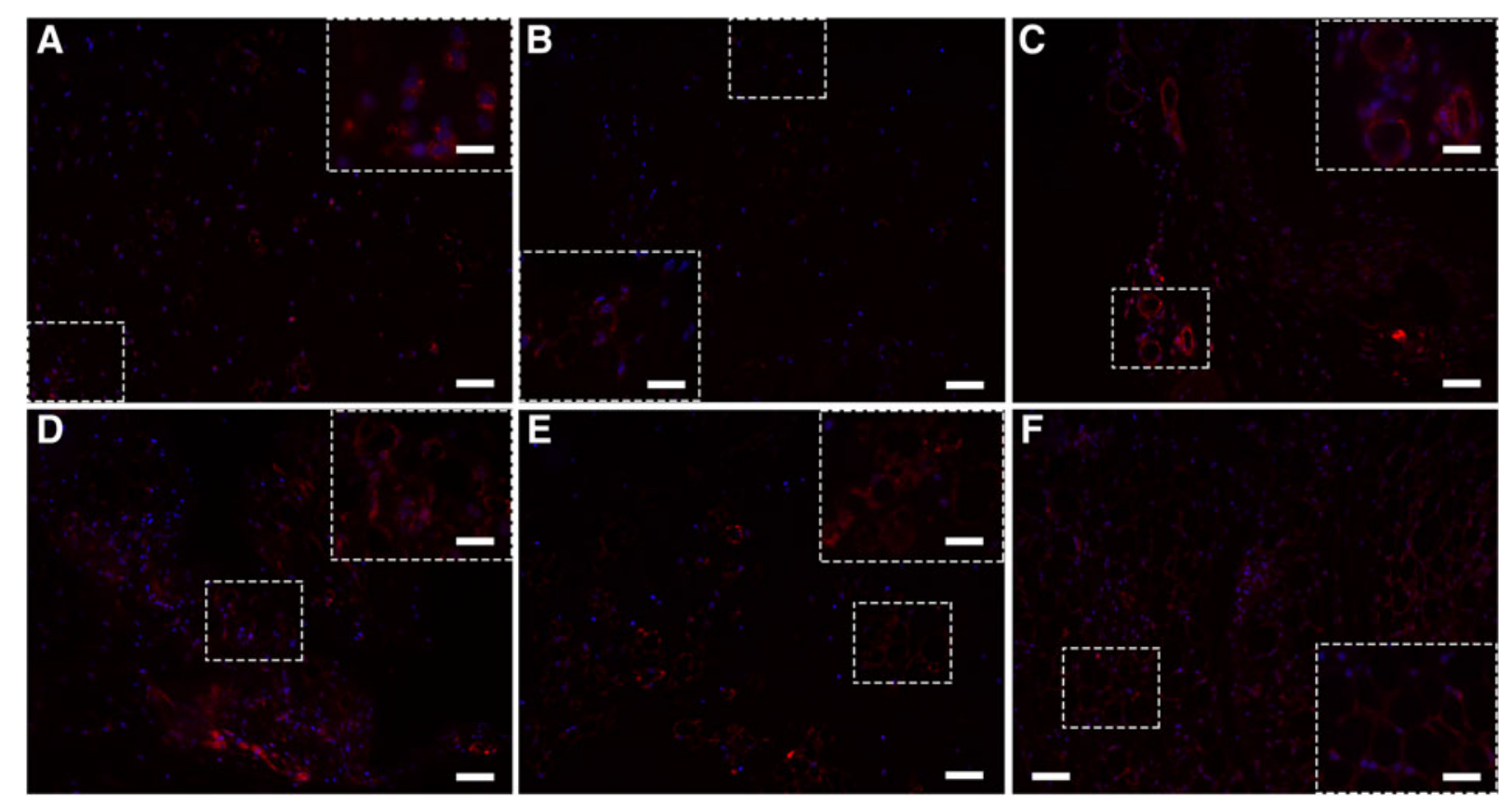

FIG. 2. Matrices were transplanted subcutaneously in naive BALB/c mice and then retrieved at 2 or 4 weeks after transplantation. Matrices were paraffin embedded; sections were stained for CD31 (red) and DAPI (blue). Vessels of matrices with $7.5 \mathrm{mM}(\mathbf{A}, \mathbf{D}), 30 \mathrm{mM}(\mathbf{B}, \mathbf{E})$, and $120 \mathrm{mM}(\mathbf{C}, \mathbf{F})$ cross-linker concentrations were visualized at 2 weeks (A, B, C) and 4 weeks (D, E, F) after transplantation. Scale bars of (A-F) are $50 \mu \mathrm{m}$; scale bars of high magnification insets are $10 \mu \mathrm{m}$.
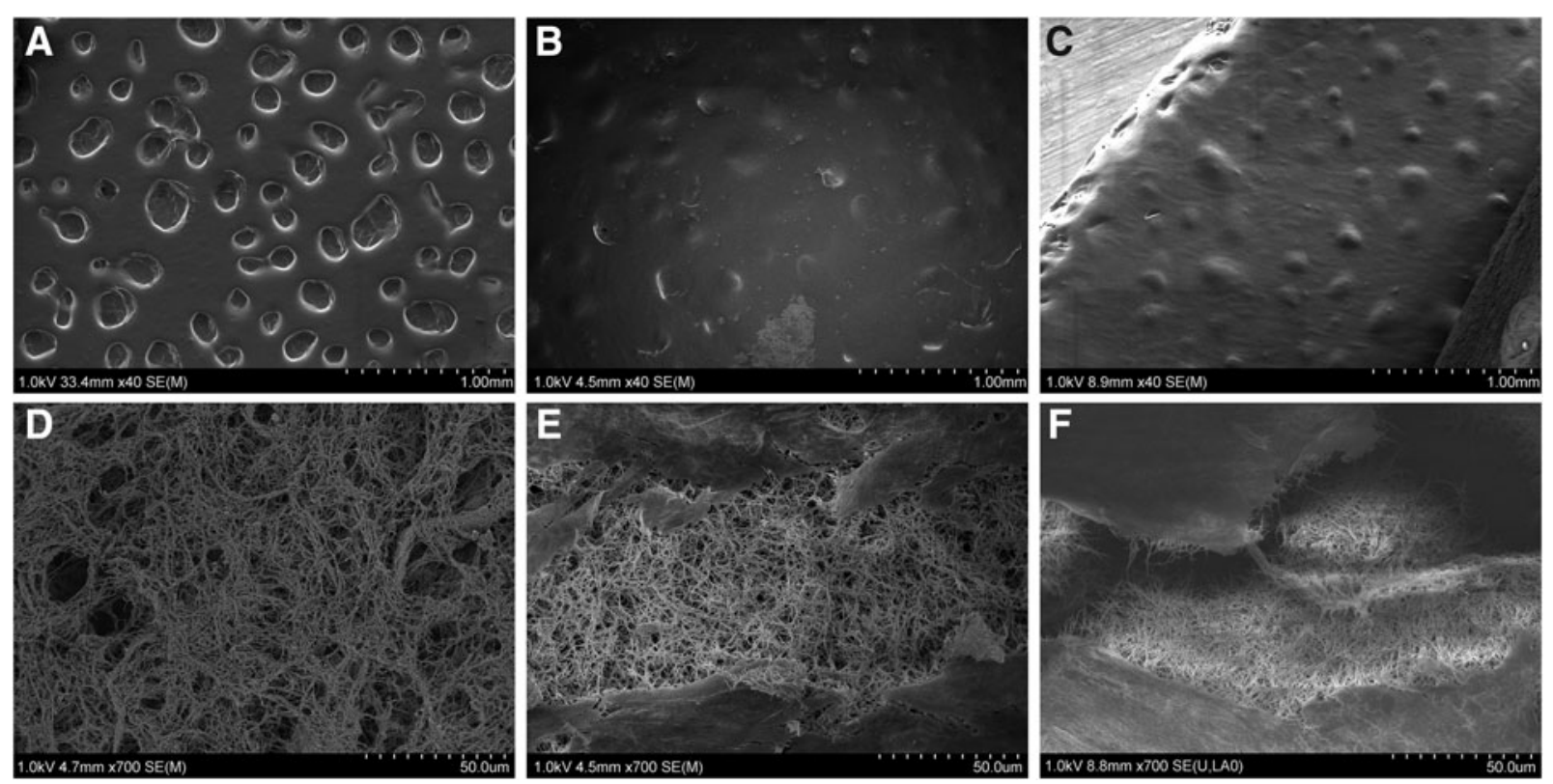

FIG. 3. The microstructures of the matrices with $7.5 \mathrm{mM}(\mathbf{A}, \mathbf{D}), 30 \mathrm{mM}$ (B, E), and $120 \mathrm{mM}(\mathbf{C}, \mathbf{F})$ cross-linker concentrations were examined using scanning electron microscopy. The surface topographies (A-C) and interior topographies (D-F) were examined. 

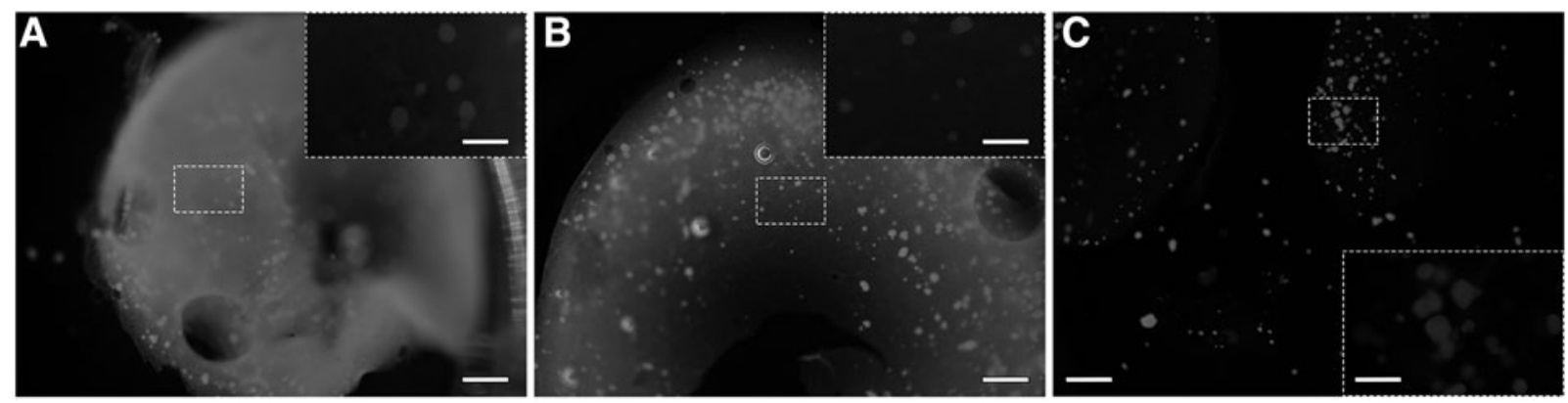

FIG. 4. NPIs embedded in matrices with $7.5 \mathrm{mM}$ (A), $30 \mathrm{mM}$ (B), and $120 \mathrm{mM}$ (C) cross-linker concentrations were cultured for 7 days, and then, dark field images were taken of the matrices in a six-well plate. Scale bars are $1.6 \mathrm{~mm}$ for $(\mathbf{A}-\mathbf{C})$; scale bars of high magnification insets are $400 \mu \mathrm{m}$. NPIs, neonatal porcine islets.

The matrix supports NPI viability at all cross-linker concentrations

After 7 days of in vitro culture with embedded NPIs, there were visible differences between the matrices with the three different cross-linking concentrations. The group with the $7.5 \mathrm{mM}$ cross-linking concentration was the most opaque and maintained sufficient mechanical integrity for manipulation. There appeared to be pores visible under light microscopy (Fig. 4A, inset). Matrices with $30 \mathrm{mM}$ cross-linking concentration had improved mechanical integrity compared to the $7.5 \mathrm{mM}$ group and were slightly more translucent (Fig. 4B). Interestingly, the addition of NPIs to the matrices with $120 \mathrm{mM}$ cross-linking concentration caused the matrices to lose mechanical integrity after 7 days such that manipulation resulted in the matrix fragmenting into multiple pieces (Fig. 4C). The matrices with $120 \mathrm{mM}$ cross-linking concentration were completely transparent. Intact NPIs were visible in all three matrix groups (Fig. 4, insets).

No TUNEL-positive cells were visible in any of the matrices (Fig. 5), indicating excellent support for the NPIs. The control NPIs cultured in the standard Ham's F10 media (Fig. 5A) had the most apoptotic cells as one or two cells per section were TUNEL positive.

\section{Discussion}

These data, combined with our previously described results, demonstrate that this collagen-based matrix has properties reproducibly tunable by the cross-linker concentration and thus could be adapted for various tissue engineering and cell delivery purposes. Our previous work has demonstrated that the matrix with
$30 \mathrm{mM}$ cross-linker concentration promotes survival and function of NPIs in vivo ${ }^{18}$; data from this study will allow further optimization of the matrix to allow adjustment of the biocompatibility of the system in a variety of situations. All matrices tested have sufficient initial mechanical strength to be easily transplanted and subsequently retrieved if necessary, which may be an important factor when delivering a xenogeneic cell product such as NPIs. ${ }^{10}$

The swelling ratio of the matrix was significantly correlated with cross-linker concentration. Controlling the swelling ratio of a hydrogel could offer control of drug release $\mathrm{e}^{23}$; this matrix could be functionalized with additional growth factors, such as vascular endothelial growth factor (VEGF), localized immunosuppressive drugs, or anti-inflammatory cytokines or drugs to protect the cell product. This could also be modified by adjusting the concentration of chondroitin-6sulfate, a glycosaminoglycan (GAG) known to bind and sequester growth factors and cytokines, ${ }^{16}$ if a specific level of cross-linker concentration was required for another aspect of biocompatibility. Insulin was diffused more quickly in matrices with a lower crosslinking concentration, supporting the rationale to adjust the cross-linker concentration as a technique for controlling drug release. In particular, the immunomodulatory and anti-inflammatory cytoprotective factors secreted by mesenchymal stem cells (MSCs) have been shown to protect islets from proinflammatory cytokines ${ }^{24}$; the matrix could have hepatocyte growth factor, fibroblast growth factor, or MSCs themselves added to further protect the islet graft. The properties of the collagen-based matrix could then be tuned to support MSC viability and function. 

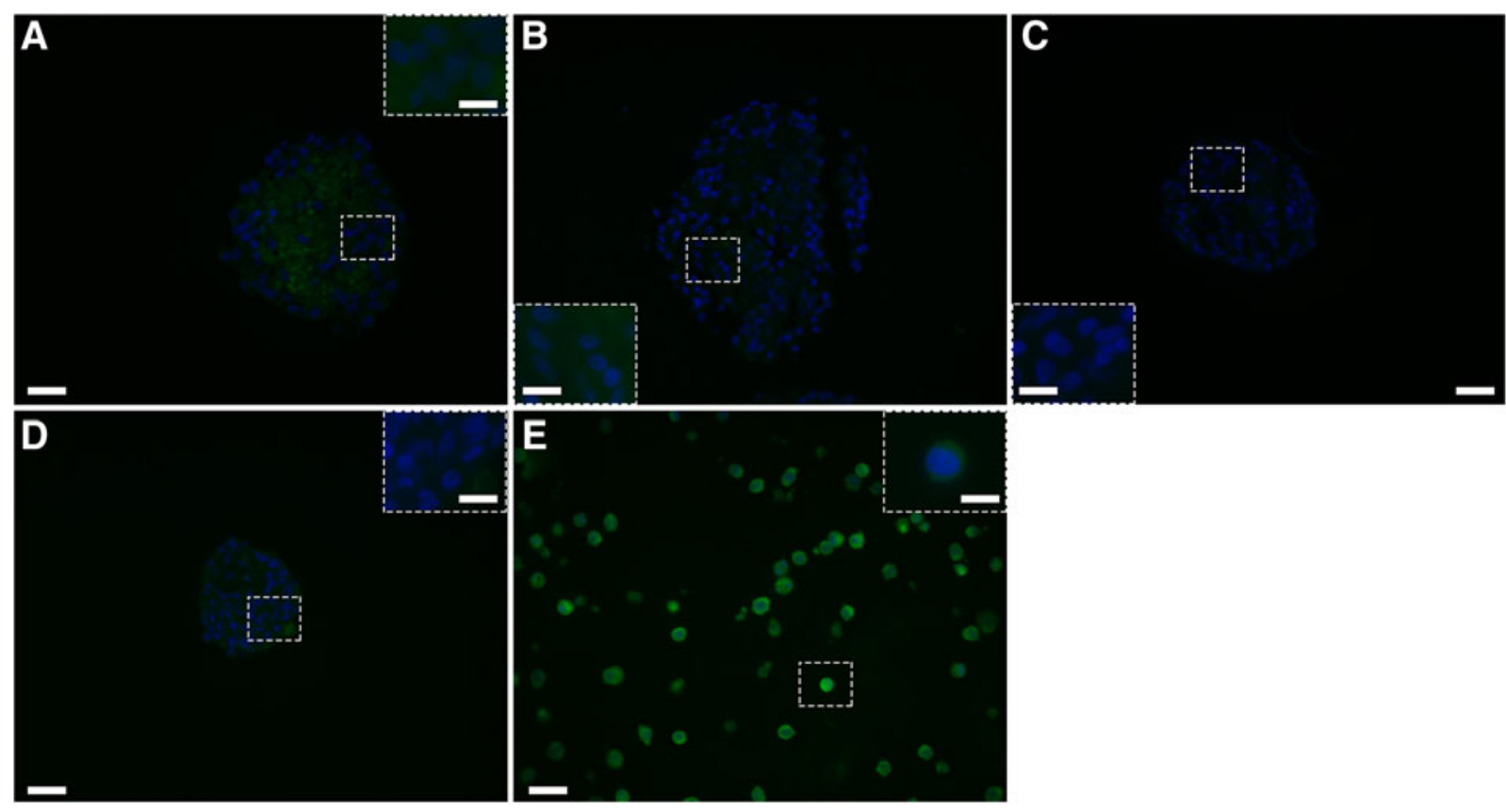

FIG. 5. NPIs embedded in matrices with $7.5 \mathrm{mM}$ (B), $30 \mathrm{mM}$ (C), and $120 \mathrm{mM}$ (D) cross-linker concentrations and in standard Ham's F10 culture media as a control (A) cultured for 7 days, then fixed and paraffinembedded; sections were stained for TUNEL and compared to TUNEL-positive controls (E). Scale bars of (A-E) are $20 \mu \mathrm{m}$ and scale bars of high magnification insets are $10 \mu \mathrm{m}$.

Matrices with higher cross-linker concentrations better resisted collagenase degradation compared to those with lower cross-linker concentrations. This is related to the swelling ratio as less fluid entering the matrix decreases the exposure to the collagenase solution. All matrices showed sufficient resistance to in vivo degradation to allow transplantation, ${ }^{15}$ but it is valuable to have the capacity to control degradation for diverse applications; for example, to match the natural healing or regeneration processes of the recipient tissues. ${ }^{25}$ The number and size of vessels also increase with cross-linker concentration, an advantageous property as these higher cross-linking concentration matrices would have a greater neovascular system to support transplanted cells in the long term. As higher crosslinker concentrations did not have cytotoxic effects on the NPIs, a matrix transplanted with a higher cross-linker concentration, transplanted without a cell population, could benefit from faster more functional vascularization of an ectopic site. However, this remains to be tested in vivo.

SEM imaging of the matrix with 7.5, 30, and $120 \mathrm{mM}$ cross-linking concentration revealed uniform micro- structure throughout the samples. Interestingly, the micropores visible on the surface of the $7.5 \mathrm{mM}$ matrix do not correlate with increased vascularization, whereas the microconvexities in the $120 \mathrm{mM}$ matrix are related to faster neovascularization in vivo. There appears to be a relationship between higher surface energy from cross-linking and the resultant geometric changes and vascularization. The geometric changes lower the surface energy of the matrices; surface energy is an important characteristic of a material that may be more important than topography for guiding cellular adhesion and proliferation. ${ }^{26}$ Although the surface energies of these matrices were not directly measured, the microconvexities of the $120 \mathrm{mM}$ cross-linking concentration matrices are likely a result of higher surface energy that allows for improved cell spreading and ameliorated vascularization. ${ }^{27,28}$ The dense interconnected structure contributes to the mechanical strength of the matrices and may facilitate cell attachment and intracellular signalling. ${ }^{29}$ Additionally, the availability of ECM proteins such as laminin and collagen can provide vital signaling cues and allow ECM receptor interactions; degradation of these proteins can provide 
substrates as well as space for angiogenesis. ${ }^{30}$ Thus, both the surface energy and topography can potentially be tuned by the cross-linking concentration to support the target cell population, for example, to encourage angiogenesis.

The rapid increase in vascularization between 2 and 3 weeks in the $120 \mathrm{mM}$ matrices is likely due to increasing porosity from biodegradation and infiltrating endothelial cells that remodel the matrix. ${ }^{31}$ SEM imaging of vascularized matrices at varying time points would be helpful in elucidating how the neovasculature forms in the center of the matrix, with or without the presence of proangiogenic factors, such as VEGF. Tuning the porosity to mimic that of the matrices at later time points could also be useful for promotion of angiogenesis. Alternatively, McFadden et al. ${ }^{32}$ demonstrated a technique for in vitro prevascularization of a collagen-GAG matrix using human umbilical vein endothelial cells and MSCs. Additionally, a matrix could be transplanted subcutaneously for longer than 4 weeks without a cell population to establish vascularization in the desired site before the delivery of the graft. As this matrix cross-links at $37^{\circ} \mathrm{C}$, a liquid scaffold could be noninvasively injected to fill the subcutaneous space. ${ }^{17,33}$ The cytotoxicity of higher cross-linker concentrations in this context requires further exploration. These could be promising approaches for engineering a matrix that can support NPI in a subcutaneous space that lacks sufficient initial vascularization to support the graft. $^{10}$

The natural ECM of the pancreas is highly complex and varies between species. ${ }^{16,34}$ Although the specific roles of the various proteins on endocrine function are not well understood, it is well known that isolated islets suffer from anoikis, a form of apoptosis. ${ }^{35}$ The lack of TUNEL-positive islet cells within the matrix supports the rationale for including collagen, laminin, GAG, and polysaccharide such as chitosan, to prevent this form of apoptosis by presenting a scaffold with similar components as the natural pancreatic ECM. ${ }^{16,35}$ Continued research on the specific interactions between NPIs and these matrix components is warranted to further ameliorate NPI survival and function.

\section{Conclusions}

Overall, these data indicate that this collagen-based matrix with the incorporation of chitosan, chondroitin-6-sulfate, and laminin is reproducible, tunable, and may be used to create a vascularized site for cell de- livery, specifically for but not limited to NPIs. This matrix offers the potential for creating a vascularized ectopic site that can be thoughtfully designed for diverse applications.

\section{Acknowledgments}

This study is supported by the Canadian Institutes of Health Research (MOP 119500) and the Juvenile Diabetes Research Foundation (Grant 117-2013-286). C.E.E. is a recipient of an Alberta Innovates Health Solutions Studentship. We thank Dr. Kunimasa Suzuki (Alberta Diabetes Institute Molecular Biology Core, University of Alberta) for assistance with the Meso Scale Discovery Assays; the University of Alberta Swine Research Centre for the neonatal piglets; and Deb Dixon for assistance with isolating the NPI. The funders had no role in study design, data collection and analysis, decision to publish, or publication of article.

\section{Author Disclosure Statement}

No competing financial interests exist.

\section{References}

1. Ryan EA, Lakey JRT, Rajotte RV, et al. Clinical outcomes and insulin secretion after islet transplantation with the Edmonton protocol. Diabetes. 2001;50:710-719.

2. Shapiro AMJ, Lakey JRT, Ryan EA, et al. Islet transplantation in seven patients with type 1 diabetes mellitus using a glucocorticoid-free immunosuppressive regimen. N Engl J Med. 2000;343:230-238.

3. Shapiro AMJ, Ricordi C, Hering BJ, et al. International trial of the Edmonton protocol for islet transplantation. N Engl J Med. 2006:355:1318-1330.

4. Street CN, Lakey JRT, Shapiro AMJ, et al. Islet graft assessment in the Edmonton protocol-implications for predicting long-term clinical outcome. Diabetes. 2004;53:3107-3114.

5. Villiger $P$, Ryan EA, Owen $R$, et al. Prevention of bleeding after islet transplantation: lessons learned from a multivariate analysis of 132 cases at a single institution. Am J Transplant. 2005;5:2992-2998.

6. Bhargava R, Senior PA, Ackerman TE, et al. Prevalence of hepatic steatosis after islet transplantation and its relation to graft function. Diabetes. 2004;53:1311-1317.

7. Markmann JF, Rosen M, Siegelman ES, et al. Magnetic resonance-defined periportal steatosis following intraportal islet transplantation-a functional footprint of islet graft survival? Diabetes. 2003;52:1591-1594.

8. Vincenti F, Larsen C, Durrbach A, et al. Costimulation blockade with belatacept in renal transplantation. N Engl J Med. 2005;353:770-781.

9. Robertson RP. Islet transplantation as a treatment for diabetes-reply. N Engl J Med. 2004;350:2104-2104.

10. Veriter S, Gianello P, Dufrane D. Bioengineered sites for islet cell transplantation. Curr Diabetes Rep. 2013;13:745-755.

11. Balamurugan AN, Gu Y, Tabata Y, et al. Bioartificial pancreas transplantation at prevascularized intermuscular space: effect of angiogenesis induction on islet survival. Pancreas. 2003;26:279-285.

12. DeVos $P$, Hillebrands JL, DeHaan BJ, et al. Efficacy of a prevascularized expanded polytetrafluoroethylene solid support system as a transplantation site for pancreatic islets. Transplantation. 1997;63:824-830.

13. Hou YT, ljima H, Takei T, et al. Growth factor/heparin-immobilized collagen gel system enhances viability of transplanted hepatocytes and induces angiogenesis. J Biosci Bioeng. 2011;112:265-272.

14. Uludag H, De Vos P, Tresco PA. Technology of mammalian cell encapsulation. Adv Drug Deliv Rev. 2000;42:29-64. 
15. Williams DF. On the mechanisms of biocompatibility. Biomaterials. 2008;29:2941-2953.

16. Cheng JYC, Raghunath $M$, Whitelock J, et al. Matrix components and scaffolds for sustained islet function. Tissue Eng Part B Rev. 2011;17:235-247.

17. Ahmadi A, Vulesevic $B$, Ruel $M$, et al. A collagen-chitosan injectable hydrogel improves vascularization and cardiac remodeling in a mouse model of chronic myocardial infarction. Can J Cardiol. 2013;29:S203-S204.

18. Ellis $C E$, Vulesevic $B$, Suuronen $E$, et al. Bioengineering a highly vascularized matrix for the ectopic transplantation of islets. Islets. 2013;5:216-225.

19. Williams DF. There is no such thing as a biocompatible material. Biomaterials. 2014;35:10009-10014.

20. McEwan K, Padavan DT, Deng C, et al. Tunable collagen hydrogels are modified by the therapeutic agents they are designed to deliver. J Biomat Sci Polym Ed. 2012;23:1467-1483.

21. McBane JE, Vulesevic B, Padavan DT, et al. Evaluation of a collagenchitosan hydrogel for potential use as a pro-angiogenic site for islet transplantation. PLoS One. 2013;8:e77538-e77538.

22. Korbutt GS, Elliott JF, Ao ZL, et al. Large scale isolation, growth, and function of porcine neonatal islet cells. J Clin Invest. 1996;97:2119-2129.

23. Lin CC, Metters AT. Hydrogels in controlled release formulations: network design and mathematical modeling. Adv Drug Deliv Rev. 2006;58:1379-1408.

24. Yeung TY, Seeberger KL, Kin T, et al. Human mesenchymal stem cells protect human islets from pro-inflammatory cytokines. PLoS One. 2012;7:e38189-e38189.

25. Nair LS, Laurencin CT. Biodegradable polymers as biomaterials. Prog Polym Sci. 2007;32:762-798.

26. Hallab NJ, Bundy KJ, O'Connor K, et al. Evaluation of metallic and polymeric biomaterial surface energy and surface roughness characteristics for directed cell adhesion. Tissue Eng. 2001;7:55-71.

27. Närhi TO. Soft Tissue Attachment on Implant Surface. In: Tissue Engineering and Regenerative Medicine: A Nano Approach. Ramalingam M, Vallittu P, Ripamonti U, Li W-J, (eds.) CRC Press: Boca Raton, FL; p. 189204; 2013.

28. Schakenraad JM, Busscher HJ, Wildevuur $\mathrm{CRH}$, et al. Thermodynamic aspects of cell spreading on solid substrata. Cell Biophys. 1988;13:75-91.

29. van Tienen TG, Heijkants RGJC, Buma $P$, et al. Tissue ingrowth polymers and degradation of two biodegradable porous with different porosities and pore sizes. Biomaterials. 2002;23:1731-1738.
30. Serbo JV, Gerecht S. Vascular tissue engineering: biodegradable scaffold platforms to promote angiogenesis. Stem Cell Res Ther. 2013;4:1-8.

31. O'Brien FJ, Harley BA, Yannas IV, et al. The effect of pore size on cell adhesion in collagen-GAG scaffolds. Biomaterials. 2005;26:433-441.

32. McFadden TM, Duffy GP, Allen AB, et al. The delayed addition of human mesenchymal stem cells to pre-formed endothelial cell networks results in functional vascularization of a collagen-glycosaminoglycan scaffold in vivo. Acta Biomater. 2013;9:9303-9316.

33. Hou QP, De Bank PA, Shakesheff KM. Injectable scaffolds for tissue regeneration. J Mater Chem. 2004;14:1915-1923.

34. Wang RN, Paraskevas S, Rosenberg L. Characterization of integrin expression in islets isolated from hamster, canine, porcine, and human pancreas. J Histochem Cytochem. 1999;47:499-506.

35. Stendahl JC, Kaufman DB, Stupp SI. Extracellular matrix in pancreatic islets: relevance to scaffold design and transplantation. Cell Transplant. 2009;18:1-12.

Cite this article as: Ellis CE, Ellis LK, Korbutt RS, Suuronen EJ, Korbutt GS (2015) Development and characterization of a collagen-based matrix for vascularization and cell delivery, BioResearch Open Access 4:1, 188-197, DOI: 10.1089/biores.2015.0007.

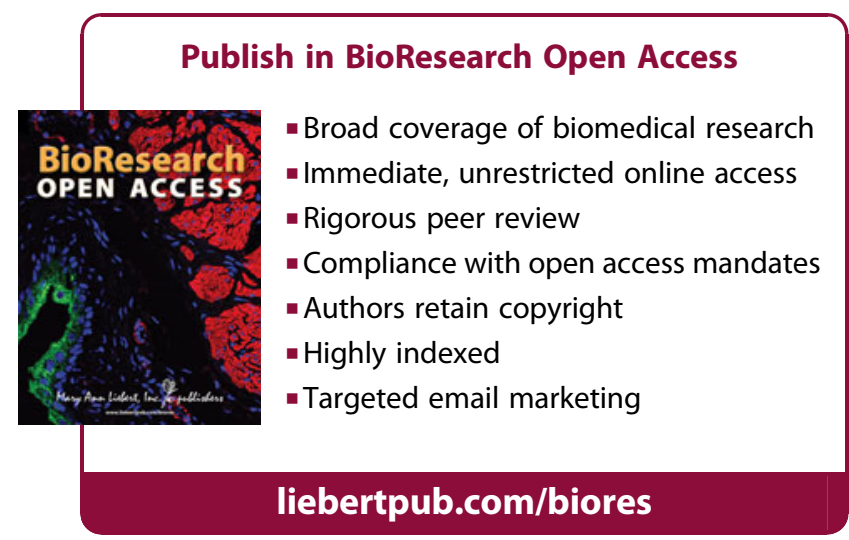

